4 Müller M, Dahmen G, Pörksen E, Geerling G, Laqua H, Ziegler A et al. Anterior chamber angle measurement with optical coherence tomography: intraobserver and interobserver variability. J Cataract Refr Surg 2006; 32: 1803-1808.

T Dada, BM Shah, SJ Bali, N Bansal, A Panda and M Vanathi

Dr Rajendra Prasad Centre for Ophthalmic

Sciences, All India Institute of Medical Sciences,

New Delhi, India

E-mail: tanujdada@gmail.com

Eye (2011) 25, 1522-1524; doi:10.1038/eye.2011.210; published online 9 September 2011

\section{Sir, \\ Abnormal corneal nerves in a patient with Lyme disease}

Lyme disease is a multiorgan disease caused by a spirochete, Borrelia burgdorferi. Although numerous ophthalmologic manifestations have been described following Lyme disease, ${ }^{1,2}$ this case describes a new feature: a bilateral corneal neuropathy.

\section{Case report}

A 69-year-old woman was referred for a suspicion of corneal dystrophy in both eyes that appeared during the last 6 months. Her past medical history was relevant for a tick bite complicated by Lyme disease 2 years ago. Despite oral antibiotic treatment (clarithromycin), she still suffered from peripheral neuropathy in the lower extremities.

She presented with complaints of decrease vision bilaterally. Best corrected visual acuity was $20 / 25$ in both eyes. Slit-lamp examination revealed irregularly enlarged corneal stromal nerves in both eyes (Figure 1). These abnormalities did not reach the central cornea and were predominantly observed in the mid-peripheral area of the right eye. Except this finding, corneal examination was normal in both eyes. No sign of inflammation of the anterior segment was observed. Examination of the posterior segment of both eyes was normal. The slight decrease in visual acuity was explained by a mild bilateral cataract. Using the Cochet-Bonnet aesthesiometer, a marked hypoesthesia was observed in the central cornea of the right eye $(3.5 \mathrm{~mm})$, whereas the corneal sensitivity was subnormal in the left eye $(5.5 \mathrm{~mm})$. Except the corneas, the sensitivity in the area innervated by the trigeminal nerves was not altered. In vivo confocal microscopy (HRT3, Heidelberg Engineering, Heidelberg, Germany) revealed enlarged stromal nerves with tortuous and abnormal branching in both eyes. Some nerve fibers showed a hyper-reflective peri-nerve infiltration (Figure 2).

\section{Discussion}

Cranial nerve involvements are well documented in early disseminated Lyme disease, ${ }^{3}$ but in the presented case, only the corneal nerves of both eyes showed alterations that were observed 2 years after the infection. In our patient, the association of distal paresthesia, suggesting axonal polyneuropathy and corneal nerves alterations, strongly supported the responsibility of Lyme disease. The mechanisms responsible for peripheral

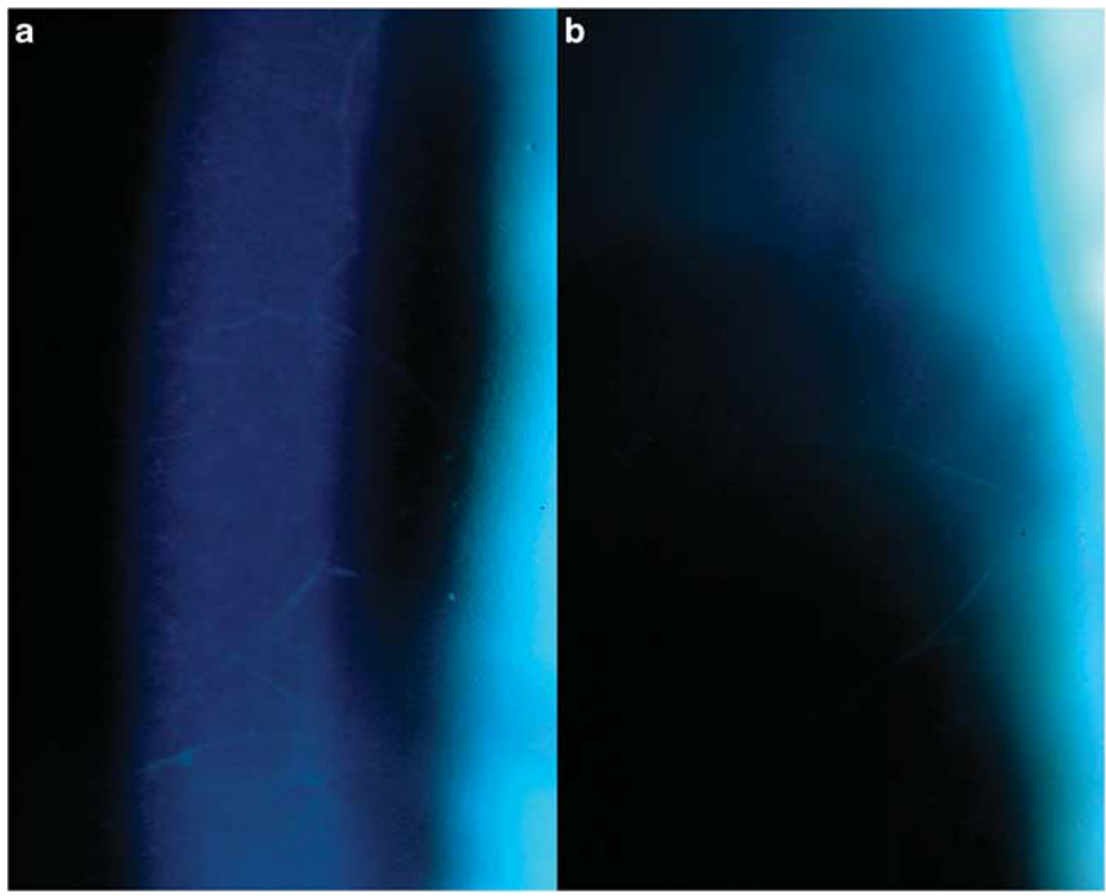

Figure 1 Slit-lamp images $(\times 20)$ of irregularly enlarged nerves within the corneal stroma of the right eye (a) and the left eye $(b)$. 

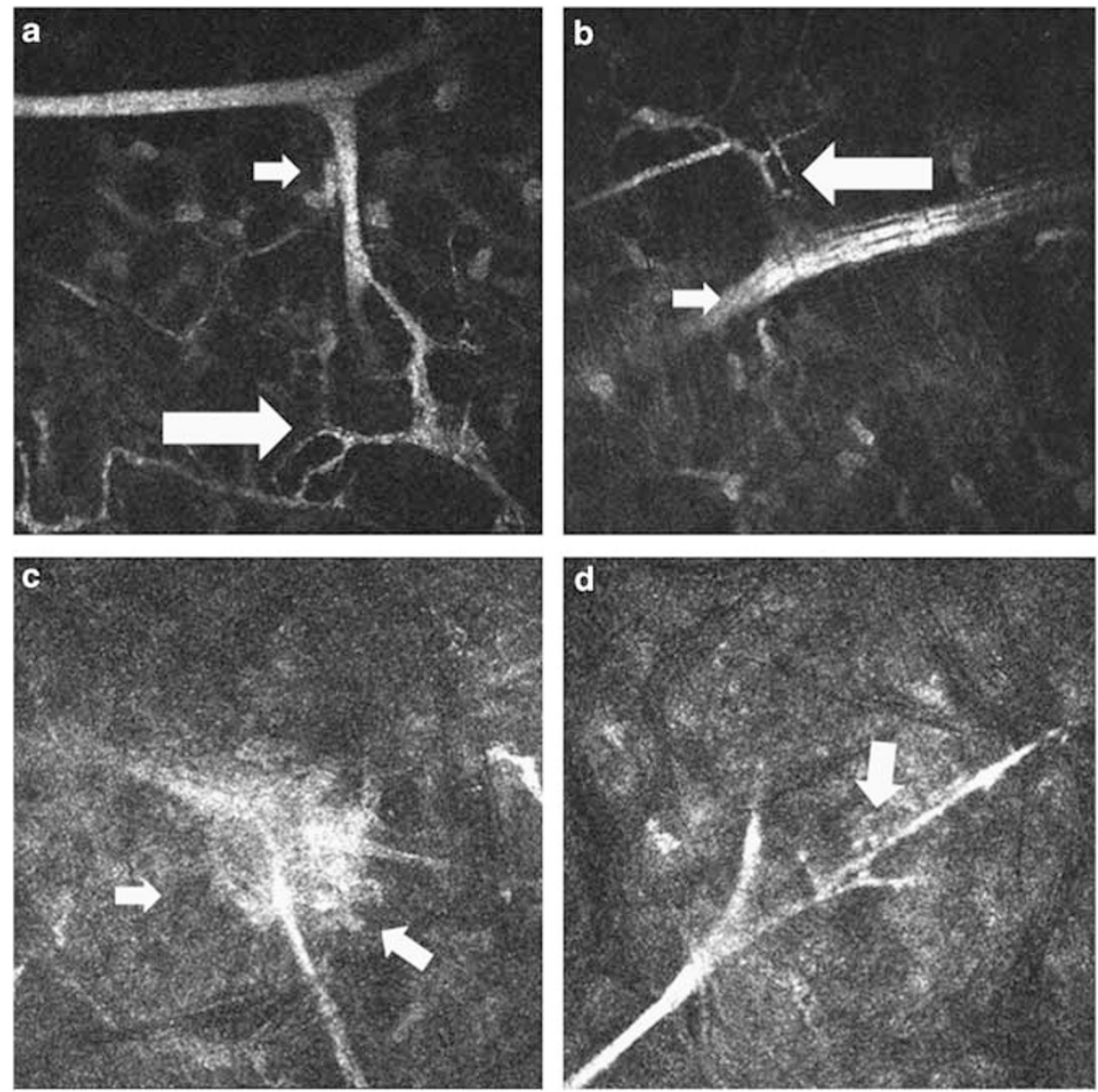

Figure 2 In vivo confocal microscopy images $(400 \mu \mathrm{m} \times 400 \mu \mathrm{m})$ of the corneas of both eyes showing corneal nerve alterations Irregular enlargements of stromal nerves (small arrows) with thin tortuous and abnormal branching (large arrows) were observed in the stroma of the right (a) and the left eye (b). Some nerves demonstrated a peri-nerve hyper-reflective infiltration (small arrows) (c, right eye and $d$, left eye).

nerve damage in Lyme disease remain unclear. Nevertheless, peripheral sensory symptoms are frequent and could appear months and years after the onset of infection even with an adapted treatment. ${ }^{3,4}$ Eye examination is not systematically performed in Lyme disease, the absence of symptoms may explain why this corneal neuropathy remained unobserved. Larger studies evaluating corneal nerves morphology and functionality in Lyme disease are now needed to confirm this finding.

\section{Conflict of interest}

The authors declare no conflict of interest.

\section{References}

1 Mora P, Carta A. Ocular manifestations of Lyme borreliosis in Europe. Int J Med Sci 2009; 6: 124-125.

2 Linna T, Mikkilä H, Karma A, Seppälä I, Petroll WM, Tervo T. In vivo confocal microscopy: a new possibility to confirm the diagnosis of Borrelia keratitis? Cornea 1996; 15: 639-640.

3 Halperin JJ. Lyme disease and the peripheral nervous system. Muscle Nerve 2003; 28: 133-143.

4 Logigian EL, Kaplan RF, Steere AC. Chronic neurologic manifestations of Lyme disease. N Engl J Med 1990; 323: 1438-1444.
X Ricaud' ${ }^{1}$, J-P Rozenbaum², S Landowski ${ }^{3}$, C Baudouin ${ }^{1,4,5,6,7}$ and A Labbé ${ }^{1,4,5,6,7}$

'Department of Ophthalmology,

Quinze-Vingts National Ophthalmology Hospital, Paris and Ambroise Paré Hospital, AP-HP,

University of Versailles Saint-Quentin

en Yvelines, Versailles, France

${ }^{2}$ Ophthalmology Clinic, Sartrouville, France

${ }^{3}$ Department of Acute Medicine, RaymondPoincaré Hospital, AP-HP, Garches and University of Versailles Saint-Quentin en Yvelines, Versailles, France

${ }^{4}$ INSERM, U968, Paris, France

${ }^{5}$ UPMC University Paris 06, UMR S 968, Institut de la Vision, Paris, France

${ }^{6}$ CNRS, UMR 7210, Paris, France

${ }^{7}$ Center of Clinical Investigations 503,

Quinze-Vingts National Ophthalmology Hospital,

Paris, France

E-mail: antoinelabbe@hotmail.com

Eye (2011) 25, 1524-1525; doi:10.1038/eye.2011.214; published online 19 August 2011 